\title{
Seizure and unilateral facial nerve paralysis in a newborn with Dandy-Walker malformation - A case report
}

\author{
S Bhakta, ${ }^{1}$ S Pal, ${ }^{2}$ A Chatterjee, ${ }^{3}$ A Pan ${ }^{4}$ \\ ${ }^{1} J u n i o r$ Resident, MD Pediatrics, ${ }^{2}$ Assistant Professor, Department of Pediatric Medicine, Medical College and \\ Hospital,Kolkatta, ${ }^{3}$ Associate Professor,Department of Pediatric Medicine,Calcutta National Medical \\ College,Kolkatta, ${ }^{4}$ Lecturer, Department of Pediatric Medicine,College of Medical Sciences, Bharatpur.
}

\section{Abstract}

The Dandy-Walker syndrome (DWS) is a rare posterior fossa malformation. It can have a varied presentation depending on the age .A newborn presenting with neonatal seizure along with unilateral facial nerve palsy is rather a rare presentation of DWS and very few such cases were reported in the past we are reporting a case of a newborn male baby presenting with neonatal seizure within $48 \mathrm{hrs}$ of birth along with right sided LMN type facial nerve palsy which on due course of investigation revealed as a case of Dandy-Walker malformation.

\section{Case report}

A 36 hours old male newborn of $2.6 \mathrm{~kg}$ weight deliverd at term by elective cessarian section presented to our NICU with repeated convulsion and depressed cry, reflex and activity. The baby was the $1^{\text {st }}$ child of a non consanguineous marriage. The baby cried immediately after birth. No history of prolonged labour,birth trauma,premature rupture of membrane,maternal fever,maternal diabetes mellitus,nonsignificant maternal drug history and antenatal period was uneventful.

The baby appeared appropriate for gestational age without any external deformity.Capillary refill time,tone,colour,fontanalle were within normal limit. Heart rate was 156/min, respiratory was rate 68/ min, head circumference was $33 \mathrm{~cm}$.

We excluded hypoglycaemia, hypocalcemia and hypomagnesemia. Convulsions were controlled by Correspondence: Dr. Subhajit Bhakta E-mail:drsubhajitbhakta@gmail.com loading and $1^{\text {st }}$ half loading of I.V phenobarbitone and no convulsion occurred thereafter.Baby was put on IV $10 \%$ dextrose,I.V Cefotaxim and Amikacin,Vit K and moist oxygen.Complete hemogram showed $\mathrm{Hb}$ $18.6 \mathrm{gm} / \mathrm{d} 1$,TLC-12600/cmm(N-40,L-55, E5),platelet count-2.1lakh/cmm.Sepsis screen was negative.Blood culture showed no growth and CSF examination revealed 2 cells(all mononuclear cells),CSF sugar $66 \mathrm{mg} / \mathrm{dl}$ and protein $82 \mathrm{mg} / \mathrm{dl}$. The baby remained depressed for first $72 \mathrm{hrs}$.

On the $4^{\text {th }}$ day of life we noted right side lower motor neurone type $(\mathrm{LMN})$ facial nerve paralysis.Ocular movement and movement of all 4 limbs appeared to be normal.USG brain on $5^{\text {th }}$ day revealed agenesis of posterior part of corpus callosum without any evidence of intracerebral or intraventricular hemorrhage. MRI brain showed - partial agenesis of corpus callosum with prominent medial borders of lateral ventricles with high 
S Bhakta et al, Seizure and unilateral facial nerve

rising $3^{\text {rd }}$ ventricle, agenesis of cerebellar vermis with $4^{\text {th }}$ ventricle directly opening into fairly large cistern magna,pituitary fossa and brain stem remaining normal.The MRI finding was suggestive of DandyWalker malformation. We did an Echocardiography to exclude associated cardiac abnormality which was essentially normal apart from a small patent foramen ovale(PFO).

We reviewed antenatal serial USG abdomen reports of the mother which didn't cite any fetal abnormality. Neurosurgery consultation was taken and they advised for routine follow up and provision of early shunt surgery.We discharged the patient with oral phenobarbitone with prognosis explained to the parents.
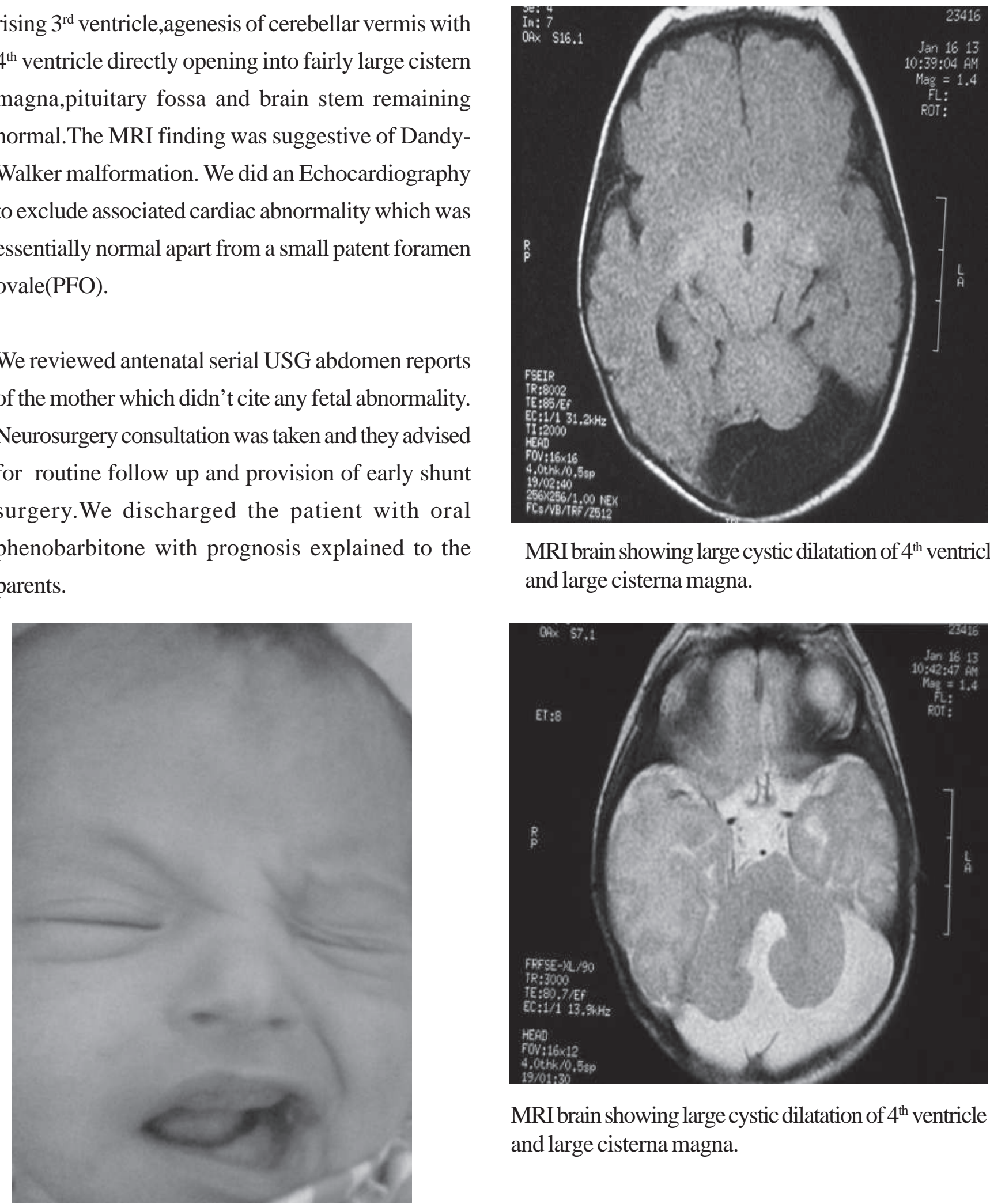

MRI brain showing large cystic dilatation of $4^{\text {th }}$ ventricle and large cisterna magna.

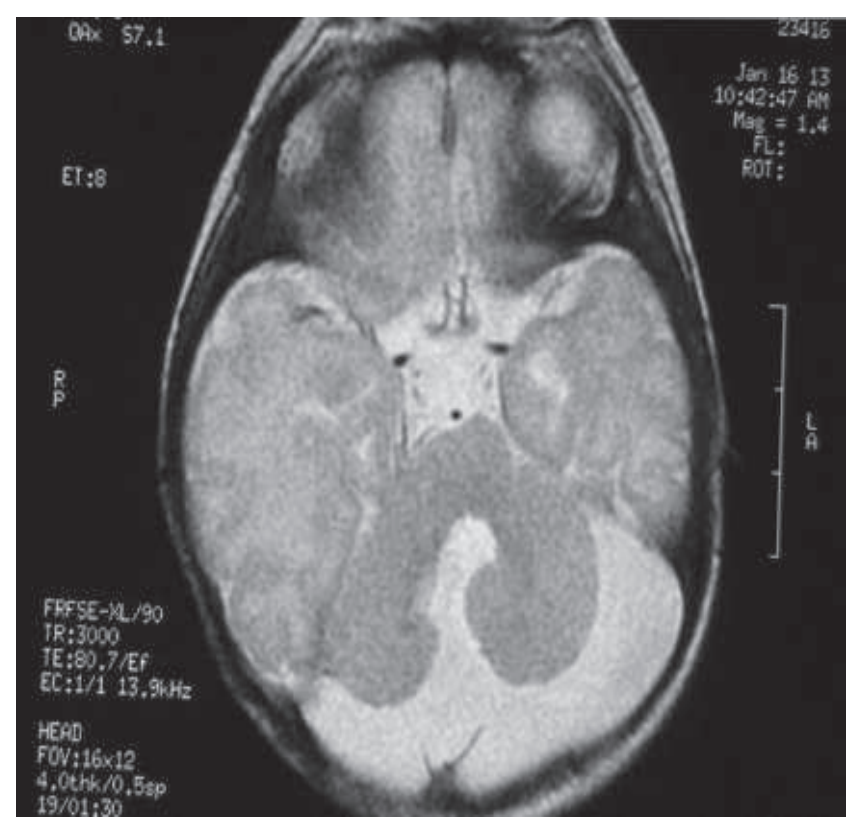

MRI brain showing large cystic dilatation of $4^{\text {th }}$ ventricle and large cisterna magna.

$4^{\text {th }}$ day old baby with right sided LMN type facial nerve palsy having dandy walker malformation. 


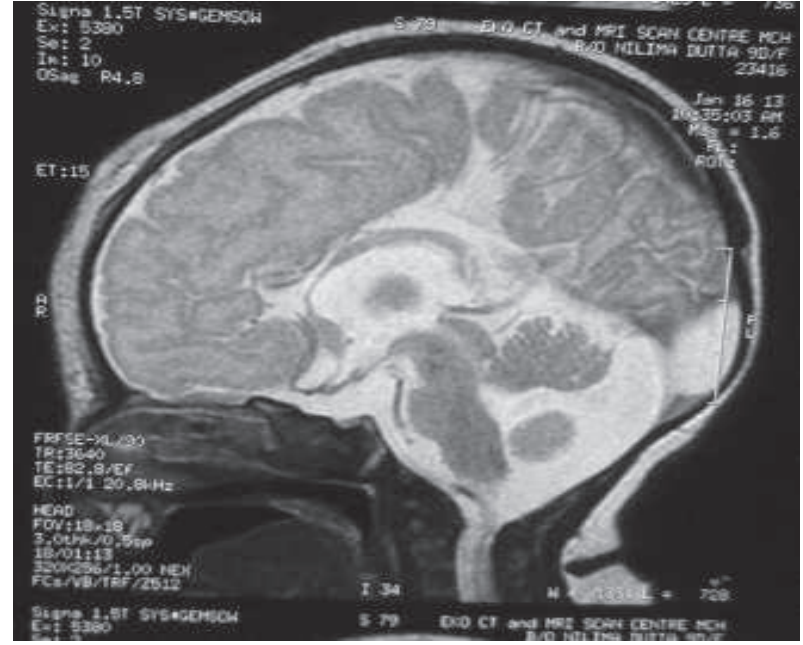

MRI brain showing agenesis of cerebellar vermis, dilated $4^{\text {th }}$ ventricle and cistern magna,partial agenesis of corpus callosum:features of dandy walker malformation.

\section{Discussion}

The DWS is a rare congenital malformation of the posterior fossa, with an incidence between 1/25000 and 1/35000 births, usually observed during the prenatal period or early infancy and more rarely in adults. ${ }^{1,2}$ The main features of the syndromes are an enlargement of the fourth ventricle, a partial or complete agenesis of cerebellar vermis, ${ }^{3}$ cyst formation near the internal base of the skull. Associated brain abnormalities are corpus callosum agenesis(17\%), occipital encephalocele(7\%), spina bifida, syringomyelia.cardiac,ocular,facial abnormalities may be associated. ${ }^{4}$

In the majority of cases of Dandy-Walker syndrome the cause is unknown, although there are a few known cases resulting from autosomal recessive genes. ${ }^{5}$ The syndrome is most often congenital but it can be acquired due to infection, chemical agents and other pre birth factors. ${ }^{6}$ Associated environmental factors include first trimester exposure to infections like rubella, cytomegalovirus, toxoplasmosis and drugs like warfarin. ${ }^{7}$

Excessive production of CSF, or failure of reabsorption due to obstruction of the passageways out of the fourth ventricle, leads to an accumulation of fluid in the brain ventricles. ${ }^{5}$ This results in the failure of parts of the cerebellum to develop correctly. A large cyst is then visible on scans of the back of the brain.

The syndrome can greatly differs in its severity and symptoms and signs depends on age of presentation. Almost $90 \%$ patients will develop hydrocephalus in the course of time.In DWS focal neurological signs are less prominent but signs and symptoms of hydrocephalus more common. ${ }^{6}$ In infancy it manifests as slow motor development with rapid enlargement of head and prominent occiput.In older children signs and symptoms of raised intracranial tension.Cerebellar disfunction, ocular,facial and neck movement abnormality also may occur. ${ }^{8}$ Neonatal seizure occurs in upto $15 \%$ cases. Dandy-Walker malformation can affect vision in variety of ways.Hearing loss is not a primary characteristic of this malformation however central hearing loss can occur due to associated corpus callosum agenesis. In our case the LMN type unilateral facial nerve palsy can be attributed to the compression of the facial colliculus at the floor of $4^{\text {th }}$ ventricle due to its cysic dilatation. Associated anomalies are cardiac $(41.7 \%)$, neurological $(33.3 \%)$, gastrointestinal $(20.8 \%)$, orthopedic $(12.5 \%)$, and genitourinary (12.5\%) abnormalities. ${ }^{9}$

Dandy Walker syndrome may be discovered before birth, by ultrasound. ${ }^{3}$ MRI is usually performed for detailed evaluation of Dandy-Walker malformation lesions and complications, after a diagnosis is 
S Bhakta et al, Seizure and unilateral facial nerve

suspected by using computed tomography (CT scan) and ultra-sound. MRI evaluation can be used antenatally to confirm the diagnosis and gain more detailed information. ${ }^{11}$

Treatment for Dandy-Walker malformation is directed to reduce the raised intracranial tension by cystoperitoneal or ventriculoperitoneal shunt or both. In smaller children with moderate hydrocephalus, cyst drainage tends to be successful.

The patients with DWS may never have normal intellectual development even when management of hydrocephalus is done early. Prognosis otherwise depends on the severity of the syndrome and associated malformations. The presence of multiple congenital defects may shorten lifespan.

Therefore, idea of this case presentation is to convey that DWS can have an early presentation since birth.Neonatal seizure with unilateral facial nerve paralysis in a newborn is rather a rare presentation of DWS.Corpus callosum agenesis as an USG brain finding may be isolated but ideally be followed by a MRI brain to exclude other brain defects like DWS.If it is diagnosed early then early shunt surgery has a better prognosis.

\section{References}

1. Hart MN, Malamud N, Ellis WG. The DandyWalker syndrome: a clinicopathological study based on 28 cases. Neurology 1972;22:771-80.

2. Hirsch JF, Pierre-Kahn A, Renier D, et al. The Dandy-Walker malformation. J Neurosurg 1984;61:515-22.

3. Klein O, Pierre-Kahn A, Boddaert N, et al. Dandy-Walker malformation: prenatal diagnosis and prognosis. Childs Nerv Syst. 2003;19(78):484-9. Epub 2003 Jul 16.

4. Imataka G, Yamanouchi H, Arisaka O.DandyWalker syndrome and chromosomal abnormalities. Congenital Anomaly (Kyoto), 2007;47(4):113-8.

5. Lavanya T, Cohen M, Gandhi SV, et al A case of a Dandy-Walker variant: the importance of a multidisciplinary team approach using complementary techniques to obtain accurate diagnostic information. British Journal Radiology 2008; 81(970):e242-5.

6. Hynd, G., Morgan, A. And Vaughn, M. Magnetic Resonance in the diagnosis of C.N.S Disorders. CIC Edizioni, pp 27-8

7. Kaplan LC (Dec 1985). "Congenital Dandy Walker malformation associated with first trimester warfarin: A case report and literature review". Teratology 32 (3): 333-7.

8. Taylor FR, Larkins MV. Headache and Chiari I malformation: clinical diagnosis, and controversies in management. Curr Pain Headache Rep 2002;6:331-7.

9. Sasaki-Adams D, Elbabaa SK, Jewells V, et al. The Dandy-Walker variant: a case series of 24 pediatric patients and evaluation of associated anomalies, incidence of hydrocephalus, and developmental outcomes. J Neurosurg Pediatr. 2008;2(3):194-9. doi: 10.3171/PED/2008/2/9/194.

10. Zimmerman RA, Bilaniuk LT; Magnetic resonance evaluation of fetal ventriculomegalyassociated congenital malformations and lesions. Semin Fetal Neonatal Med. 2005;10(5):429-43. 